\section{Analysis of White Blood Cell Segmentation Techniques and Classification Using Deep Convolutional Neural Network for Leukemia Detection \\ ${ }^{1}$ Prof. Snehal Laddha, ${ }^{2}$ Prof. Smita Daware \\ Assistant Professor, Department of Electronics Engineering, Shri. Ramdeobaba College of Engineering and Management, Nagpur, India \\ Email: *laddhasv2@rknec.edu,dawaresg@rknec.edu

\begin{abstract}
Leukemia is a blood cancer which results due to inundation of abnormal white blood cells in human. Digital pathology is a dynamic condition that empowers extraction of precise and detailed information of amount and condition of WBCs from patient's blood smear. Digital pathology methods along with machine learning is gaining importance speedily as a proven and essential technology for investigation of new features of leukemia patients histological information, providing the reduced laboratory expenses, improved operational efficiency, diagnosis. This paper highlights emerging methods to automatically diagnose leukemia from cytological-histological \& morphological analyses. In this study, we have performed comparative analysis of white blood cells segmentation techniques and evaluated the performance of pretrained deep CNN with multiclass models for Support Vector Machine (ECOC) as feature extractors towards classification of WBC to assist in improved screening of leukemia with classification accuracy of $93.94 \%$.
\end{abstract}

\title{
Keywords
}

Blood Cancer, Microscopic Images, White Blood Cells, Blood Smear, Acute Leukemia, Machine Learning, Classification, Convolutional Neural Network

\section{Introduction}

Blood cancer influences the creation and capacity of platelets in human. Leukemia is one of the types of blood cancer that begins from bone marrow, where blood is produced [1]. Stem cells mature and develop into three types of blood cells: redblood cells, white blood cells, or platelets. In blood cancer, the healthy blood cell development process is hindered by uncontrolled progress of an unusual type of blood cell. These abnormal blood cells, prevent blood from fighting off infections or preventing serious bleeding. The visual investigation of peripheral blood samples is a vital test in the methods for the identification of leukemia [24]. There are four categories of leukemia [2][3] shown in Fig. 1.

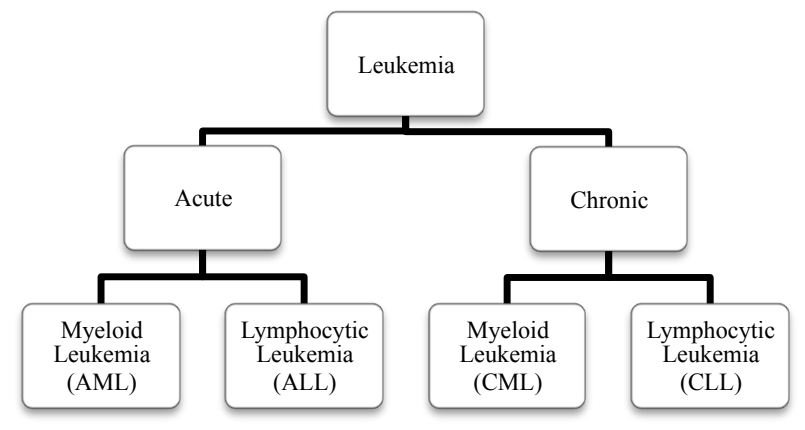

Figure 1. Types of Leukemia

Leukemia is the most widely recognized cancer in young children those ages under 15 years and adults over age of 65 years. Early and quick detection of leukemia is important for exact treatment. A pathologist's qualitative decision bolstered by a mechanized quantitative decision tool will be helpful to improve the prognostic capacity of histologicalcytological investigations by providing new diagnostic information which is not visible to the pathologist by naked eye. As histological-cytological analysis is the most proficient way for primary screening of leukemia patients, despite everything it stays as a standard leukemia analysis method.

\section{Materials and Methods}

Statistical analysis of blood smear images which includes number of RBCs, WBCs and platelets and detailed morphological features can assist a pathologist to diagnose leukemia. Blood smear images are large in size; to perform 
analysis on image first they need to be resized. Preprocessing is to be done prior to apply segmentation algorithms. Preprocessing involves image denoising, image enhancement, contrast enhancement. Then cells are detected and boundaries are traced using segmentation techniques for morphological analysis. Typical image processing steps for WBC segmentation are as shown in figure 2.

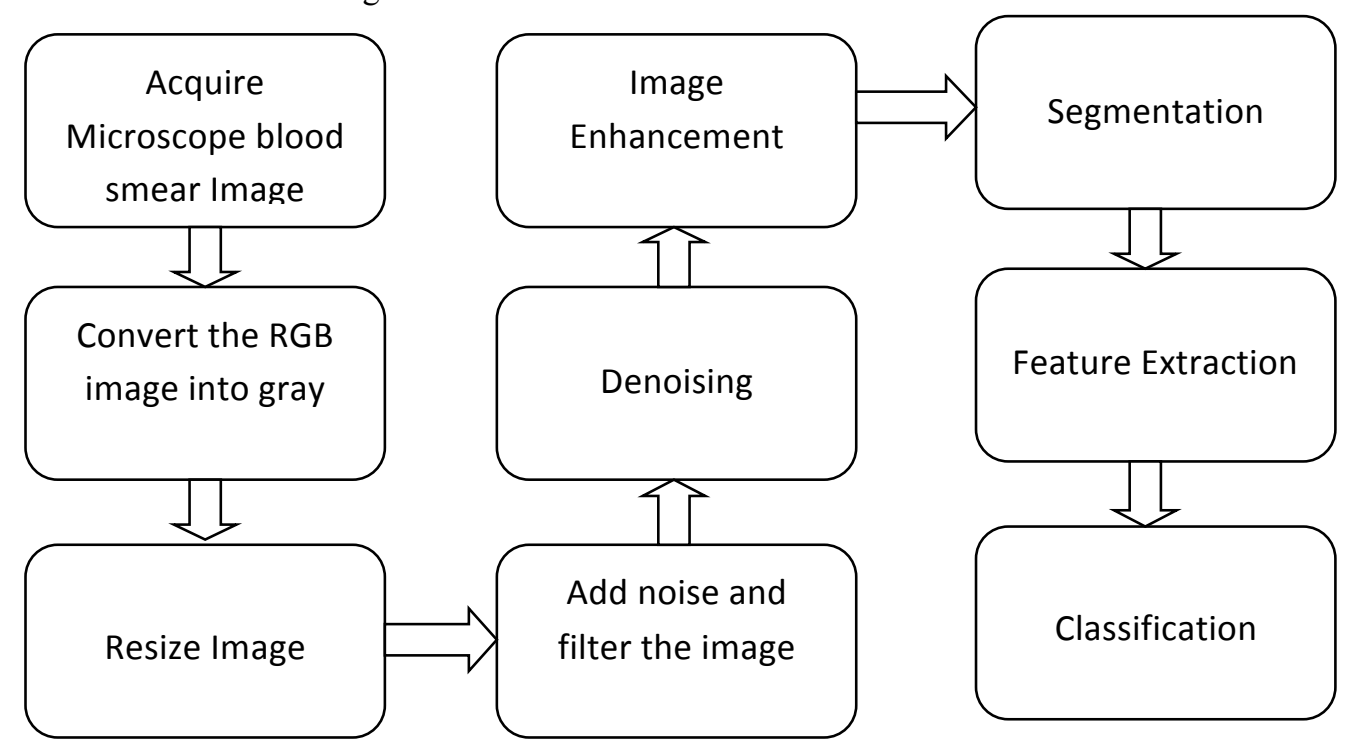

Figure 2. Image Processing Steps for Leukemia Detection From Blood Smear Images

\section{Segmentation Techniques:}

Leukemia can be detected based on the quantitative measures and size of WBCs in the blood. Identification of individual cells from microscopic images can be done by different segmentation algorithms (Table 1); below mentioned algorithms can recognize overlapping cells and can also divide each WBC into three categories such as: cell membrane, nucleus, and cytoplasm. Followed with segmentation, metrics, cell and nucleus size can be extracted from the WBCs. Figure 3 shows preprocessing of microscopic blood image and comparative analysis of different segmentation techniques.

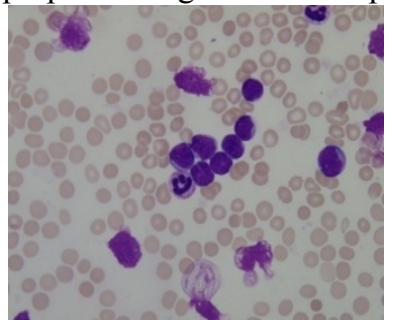

(a)

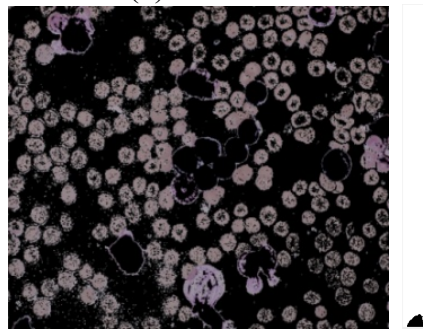

(e)

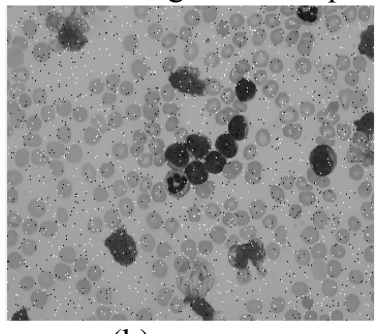

(b)
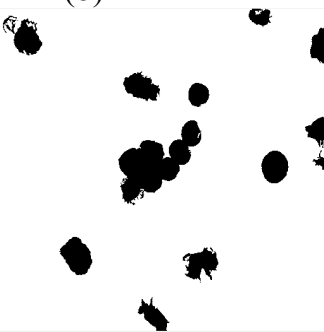

(f)

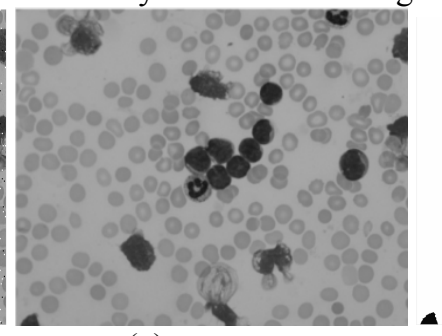

(c)

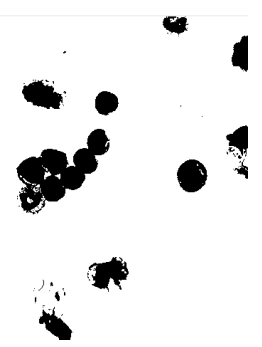

(g)

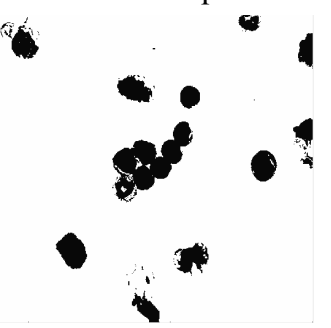

(d)

Figure 3. Results of Image Preprocessing and Segmentation Cases: a) RGB Image, b) Salt and Pepper Noise, c) Filtered and Denoised Image, d) Fuzzy C Means Segmented Image, e) Lab Color Space K Means Clustering Showing Objects in Cluster 1, f) Thresholding Opening Closing by Marker Controlled Watershed Segmentation, g) Global Image Threshold Using Otsu's Method. 


\begin{tabular}{|l|l|l|l|}
\hline $\begin{array}{l}\text { S. } \\
\text { No. }\end{array}$ & References & Segmentation Algorithms & Accuracy \\
\hline 1 & Dr. T. Karthikeyan et. al. 2017[6] & $\begin{array}{l}\text { Fuzzy K Means } \\
\text { Fuzzy C Means }\end{array}$ & $\begin{array}{l}83 \% \\
90 \%\end{array}$ \\
\hline 2 & Fabio Scotti et. al 2006 [25] & $L^{*} a^{*} b^{*}$ color space method & $92 \%$ \\
\hline 3 & Ghane N, Vard A, et. al. 2017 [4] & Thresholding & $92.07 \%$ \\
\hline 4 & Yan Li et al. 2016 [5] & Dual-Threshold Method & $97.85 \%$ \\
\hline
\end{tabular}

Table 1. Quantitative Comparison of Segmentation Algorithms in Terms of Accuracy

\section{Classification of Cytology:}

At the point when a little measure of information is accessible, traditional element designing based machine learning calculations give genuinely precise forecasts [9]. Classification of tissue types from microscopic images can be done computationally; identified cells and tissues from the images needs to be converted into a vector of features. Regular machine learning algorithms separate measurements from pictures in view of a human designing procedure that requires area learning $[12,13]$. After segmentation of WBC from the image, features are extracted either from the whole WBC or separately as Eosinophils, Baspohils, Neutrophils, Lymphocytes and Monocytes. Although, this analysis gives helpful data however it isn't adequate for the diagnosis of blood cancer. In addition to this, characteristics of multicellular gatherings should be considered [10]. Table 2 demonstrates cell-based morphometric investigation to recognize significant leukemia composes and extract features from image and methodology involved. Kazemi et al. 2016 [11] Performed color segmentation and identified irregularity, nucleus-cytoplasm ratio, shape, color, Hausdorff dimension and texture features from the entire nucleus in the whole images containing multiple nuclei using SVM. In [12] Ravikumar et al presented method which detects the majority of WBCs, using Extreme Learning machine and the fast relevance vector machine (Fast-RVM) giving strong flexibility and high computational proficiency. The Fast RVM achieves better and more adjusted characterization for individual classes also, with a low preparing time contrasted with ELM. Subrajeet et al. [15] used SCM clustering to segment every lymphocyte picture into its individual core and cytoplasm locales. Amid highlight extraction, 44 features are extracted from segmented nucleus and cytoplasm of every lymphocyte sub pictures. Agaian et al [13] used local binary pattern automatically detects and segments AML in blood smears 80 blood smear images were tested and obtained $98 \%$ precision for the localization of the lymphoblast cells and to isolate it from the subimages and complete images. Performance of HD in the wake of utilizing LBP expanded the classifier execution by $4 \%$ also extracted the edges of the nuclei of the myleoblast in a very pronounced manner. Jakkrich Laosai et al [27], Grouped leukocytes and sort them into 2 noteworthy composes which are lymphoid stem cells and myeloid stem. The technique has been assessed utilizing KMeans clustering. Features extracted from the fragmented cytoplasm and nucleus, are inspired by the visual signals of shape and surface with the highest performance of $92 \%$ using SVM. Several methods for leukemia detection have been proposed in past. Table 2 shows some of the classification techniques of the AML and ALL.

\begin{tabular}{|l|l|l|l|}
\hline References & Method & Classifier & Accuracy (\%) \\
\hline $\begin{array}{l}\text { Mohaprata et al. } \\
(2011) \text { [36] }\end{array}$ & $\begin{array}{l}\text { Fuzzy based blood image segmentation for } \\
\text { automated leukaemia detection }\end{array}$ & SVM & 93.00 \\
\hline $\begin{array}{l}\text { Putzu and Ruberto, } \\
(2013)[33]\end{array}$ & $\begin{array}{l}\text { White blood cells identification and classification } \\
\text { from leukaemia blood image }\end{array}$ & SVM & 92 \\
\hline $\begin{array}{l}\text { Mohapatra et al. } \\
(2014)[15]\end{array}$ & $\begin{array}{l}\text { An ensemble classifier system for early diagnosis } \\
\text { of acute lymphoblastic leukemia in blood } \\
\text { microscopic images }\end{array}$ & $\begin{array}{l}\text { Ensemble (RBFN } \\
+ \text { SVM + MLP + } \\
\text { KNN + NB) }\end{array}$ & 94.73 \\
\hline $\begin{array}{l}\text { Putzua et al., (2017) } \\
\text { [33] }\end{array}$ & $\begin{array}{l}\text { Leucocytes classification for leukemia detection } \\
\text { using image processing techniques }\end{array}$ & SVM & 92 \\
\hline $\begin{array}{l}\text { Karthikeyan and } \\
\text { Poornima, (2017) [6] }\end{array}$ & $\begin{array}{l}\text { Microscopic image segmentation using fuzzy C- } \\
\text { means for leukaemia diagnosis }\end{array}$ & SVM & 90 \\
\hline
\end{tabular}

Table 2. Quantitative comparison of Classification algorithms in terms of Accuracy

At the point when a lot of data involved, a profound deep learning approach can be used. Deep learning can self-find new, progressive highlights in images (feature learning) permitting better pattern recognition for classification. Deep learning methods outperform over previous conventional machine learning approaches and can achieve higher accuracy [16]. Deep learning network design is dependent on the input dataset and classification target. For pattern recognition and medical image analysis Convolutional neural networks $(\mathrm{CNNs})$ are the well suited. To identify and analyze distinctive cancers, including leukemia cells CNNs have been effectively applied to feature learning. For white blood cell, lymphocyte detection and lymphoma subtype classification deep learning strategies have been utilized [8][17][34]. 


\section{Transfer Learning Using AlexNet:}

A convolutional neural network (CNN) is a specific deep learning architecture suitable for image recognition. A CNN has input layer, output layer and multiple hidden layers (convolutional layers, pooling layers, fully connected layers and normalization layers). The performance of CNN models for natural image classification received another boost by the introduction of ImageNet by Alex Krizhevsky, thus also known as AlexNet, in 2012[30]. AlexNet is considered a breakthrough application of CNNs to multi-categorical classification. We have used a fine-tune pretrained AlexNet convolutional neural network to perform classification of Blood smear images. Network takes an image as input and outputs a label for the object in the image together with the probabilities for each of the object categories. In transfer learning a network can be trained to classify images according to applications. Here we have changed last three layers of the pretrained network to classify different categories of WBCs for leukemia detection.
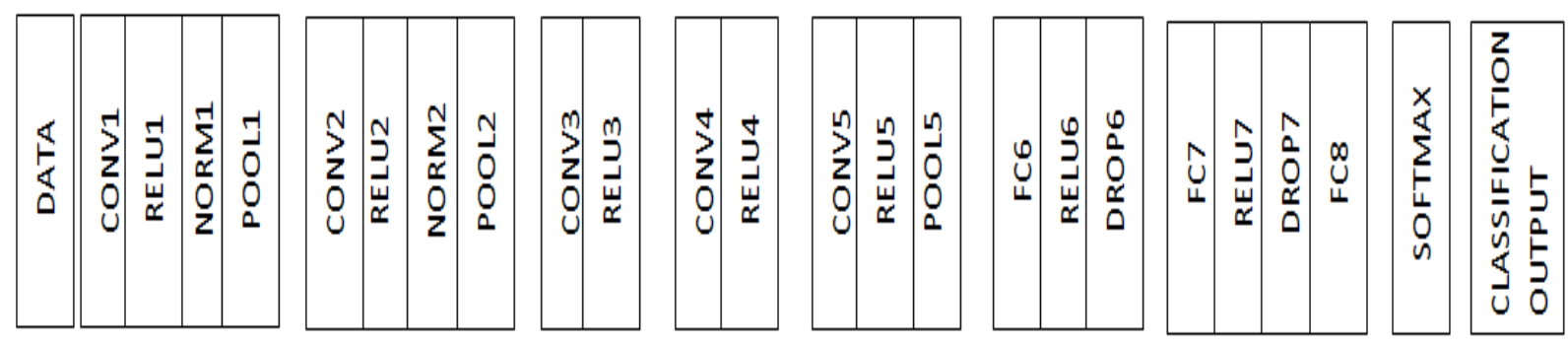

Figure 4. Pretrained Alexnet Model

\section{CNN Architecture for Blood Smear Image Classification:}

The input to the model constitutes segmented cells of $227 \times 227 \times 3$ images with zero center normalization. The proposed $\mathrm{CNN}$ has five convolutional layers and four fully connected layers. The learning process can be enhanced by using combination of convolutional/rectified linear units (ReLU) and proper weight initialization [31]. Fully connected layer Max-pooling layers with a pooling window of $3 \times 3$ and 2 pixel strides follow the convolutional layers for summarizing the outputs of neighboring neuronal groups in the feature maps. Alexnet model is shown in figure 4.

In this paper we have modified a pretrained convolutional neural network with multiclass models for Support Vector Machine (ECOC) for classification of WBC into different categories for leukemia detection. We have used 108 images from ALL-IDB [2] dataset for our research work. Figure 3 shows architecture of Alexnet with 25X1 layers. We have used Transfer learning technique where we took a pretrained CNN and utilized it as a beginning stage to take in another undertaking of WBC classification. We have selected this method as we have less data and transfer learning works faster on small data set. We have provided datasets of five different categories of WBCs as Eosinophils, Baspohils, Neutrophils, Lymphocytes and Monocytes out of them $70 \%$ of images were used for training and $30 \%$ were used for validation.

\section{Results and Discussion}

In this paper we have discussed image analysis techniques for quantitative examination of blood smear images for leukemia detection as it is one of the efficient and cost effective methods. We have reviewed different segmentation and classification techniques using machine learning to provide supervised learning to assist pathologist to diagnose leukemia at early stage. We have proposed an algorithm by modifying pretrained deep convolutional neural network Alexnet for classification of WBCs, using this we could achieve accuracy of $93.94 \%$. Results of classification of different categories of WBC images are shown in figure 5.

Deep learning approach works best on large data which could provide higher accuracy for classification. In future we would to adopt technique for leukemia detection using deep learning to acquire a new set of morphological features with higher accuracy using a large dataset. 

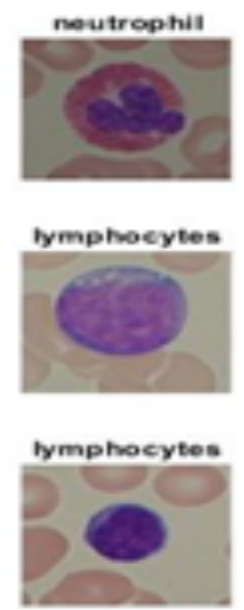
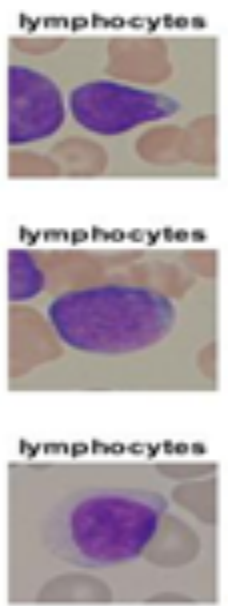
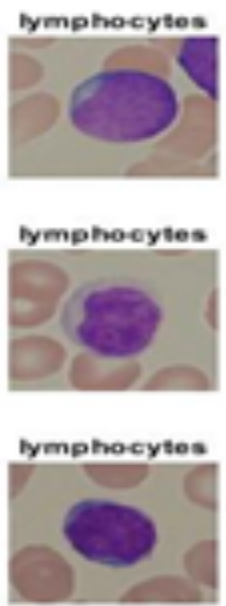

\section{Dataset :}

Figure 5. Classification Results of WBC Images into Different Categories

For this research work we have used ALL-IDB blood cell dataset [24]. ALL-IDB is public and freely available dataset of microscopic blood images, which is especially developed for the evaluation and comparison of blood cell segmentation and classification of leukemia. It has 108 JPG format images with 24 bit color depth, resolution $2592 \times 1944$.

\section{Conclusion}

In this work we have proposed an innovative method for a classification of White blood cells from microscopic images, as support for detection of leukemia. The results obtained shows that the proposed method is able to classify the white blood cells present in the blood smear image with the accuracy of $93.94 \%$ by modifying a pretrained deep convolutional neural network.

\section{Acknowledgement:}

We extend our sincere thanks to Fabio Scotti, Department of Information Technology, and University of Milan, Italy for making dataset ALL-IDB of Peripheral Blood samples of normal individuals and Leukemic Patients available for research.

\section{References}

\section{Paper in a Journal:}

[1] The American Society of Hematology, www.hematology.org

[2] M.J. Horner, L.. Ries, M. Krapcho, N. Neyman, R. Aminou, N. Howlader, S. Altekruse, E.J. Feuer, L. Huang, A. Mariotto, B.A. Miller, D.R. Lewis, M.P. Eisner, D.G. Stinchcomb, B.K. Edwards (eds), SEER Cancer Statistics Review, 1975-2006, National Cancer Institute Bethesda, MD, http://seer.cancer.gov/csr/1975_2006

[3] S. Faderl, H.M. Kantarjian, M. Talpaz, Z. Estrov, Clinical Significance of Cytogenetic Abnormalities in Adult Acute Lymphoblastic Leukemia, The Journal of The American Society of Hematology, Washington, USA, Vol. 91, No. 11, 1998.

[4] Segmentation of White Blood Cells From Microscopic Images Using a Novel Combination of K-Means Clustering and Modified Watershed Algorithm, Ghane N, Vard A, Talebi A, Nematollahy P, Journal of Medical Signals and Sensors, 2017 Apr-Jun; 7(2): 92-101

[5] Yan Li, Rui Zhu, Lei Mi, Yihui Cao, and Di Yao, "Segmentation of White Blood Cell from Acute Lymphoblastic Leukemia Images Using Dual-Threshold Method," Computational and Mathematical Methods in Medicine, vol. 2016, Article ID 9514707, 12 pages, 2016.

[6] Microscopic Image Segmentation Using Fuzzy C Means For Leukemia Diagnosis, Dr.T.Karthikeyan, N.Poornima, International Journal of Advanced Research in Science, Engineering and Technology, Vol. 4, Issue 1, January 2017

[7] Daniel Graves and Witold Pedrycz Fuzzy C-Means, Gustafson Kessel FCM, and Kernel Based FCM: A Comparative Study Analysis and Design of Intelligent Systems using Soft Computing Techniques , Springer, 2007.

[8] Janowczyk A, Madabhushi A. Deep learning for digital pathology image analysis: A comprehensive tutorial with selected use cases. Journal of Pathology Informatics. 2016;7:29

[9] Shen D, Wu G, Suk H-I. Deep learning in medical image analysis. Annual Review of Biomedical Engineering. 2017;19:221-248

[10] Rodak BF, Carr JH. Clinical Hematology Atlas. St. Louis, Missouri, USA: Elsevier Saunders; 2013 
[11] Kazemi F, Naiafabadi TA, Araabi BN. Automatic recognition of acute Myelogenous leukemia in blood microscopic images using K-means clustering and support vector machine. Journal of Medical Signals and Sensors. 2016;6(3):183-193

[12] Ravikumar, S., Image segmentation and classification of white blood cells with the extreme learning machine and the fast relevance vector machine. Artificial Cells, Nanomedicine, and Biotechnology, 1-5. doi:10.3109/21691401.2015.1008506

[13] Agaian, S., Madhukar, M., \& Chronopoulos, A. T. (2014). Automated Screening System for Acute Myelogenous Leukemia Detection in Blood Microscopic Images. IEEE Systems Journal, 8(3), 995-1004. doi:10.1109/jsyst.2014.2308452

[14] Pan, C., Park, D. S., Yang, Y., \& Yoo, H. M. (2011). Leukocyte image segmentation by visual attention and extreme learning machine. Neural Computing and Applications, 21(6), 1217-1227. doi:10.1007/s00521-011-0522-9

[15] Mohapatra, S., Patra, D., \& Satpathy, S. (2014). An ensemble classifier system for early diagnosis of acute lymphoblastic leukemia in blood microscopic images. Neural Computing and Applications, 24(7-8), 1887-1904. doi:10.1007/s00521-013-1438-3

[16] LeCun Y, Bengio Y, Hinton G. Deep learning. Nature. 2015; 521:436-444

[17] Zhao J, Zhang M, Zhou Z, Chu J, Cao F. Automatic detection and classification of leukocytes using convolutional neural networks. Medical \& Biological Engineering \& Computing. 2017;55(8):1287-1301

[18] Snehal V. Laddha, Comparative analysis of white blood cell segmentation techniques for Leukemia detection, International Journal of Latest Trends in Engineering and Technology,Volume 10, Issue 1 - March 2018, 138-141

[19] Rezatofighi, S.H., Soltanian-Zadeh, H.: Automatic recognition of five types of white blood cells in peripheral, Computerized Medical Imaging and Graphics, Volume 35, Issue 4, June 2011, Pages 333-343

[20] Awad M., Khanna R. (2015) Support Vector Machines for Classification. In: Efficient Learning Machines. Apress, Berkeley, CA

[21] Pan, S. J. \& Yang, Q. A survey on transfer learning. IEEE Trans. Knowl. Data Eng. 22, 1345-1359 (2010)

[22] Putzua L., Caoccib G., Rubertoa C. D. Leucocyte classification for leukemia detection using image processing techniques. Artificial Intelligence in Medicine. 2017;78:1-72.

[23] Khajehpour H, Dehnavi AM, Taghizad H, Khajehpour E, Naeemabadi M. Detection and Segmentation of Erythrocytes in Blood Smear Images Using a Line Operator and Watershed Algorithm. Journal of Medical Signals and Sensors. 2013;3(3):164-171.

Paper in a Conference Record:

[24]ALL-IDB: The Acute Lymphoblastic Leukemia Image Database For Image Processing, Ruggero Donida Labati, Vincenzo Piuri, Fabio Scotti, 2011 18th IEEE International Conference on Image Processing

[25] F. Scotti, "Robust Segmentation and Measurements Techniques of White Cells in Blood Microscope Images", Proceedings of the IEEE Instrumentation and Measurement Technology Conference, pp. 43-48, 2006.

[26] Nancy M. Salem, Segmentation of White Blood Cells from Microscopic Images using K-means Clustering, $31^{\text {st }}$ National Radio Science Conference (NRSC2014) April 28 - 30, 2014

[27] Laosai, J., \& Chamnongthai, K. (2014). Acute leukemia classification by using SVM and K-Means clustering. 2014 International Electrical Engineering Congress (iEECON). doi:10.1109/ieecon.2014.6925840

[28] Mohammed, E. A., Far, B. H., Mohamed, M. M. A., \& Naugler, C. (2013). Application of Support Vector Machine and k-means clustering algorithms for robust chronic lymphocytic leukemia color cell segmentation. 2013 IEEE 15th International Conference on e-Health Networking, Applications and Services (Healthcom 2013). doi:10.1109/healthcom.2013.6720751

[29] E. A. Mohammed, M. M. A. Mohamed, C. Naugler and B. H. Far, "Chronic lymphocytic leukemia cell segmentation from microscopic blood images using watershed algorithm and optimal thresholding," 2013 26th IEEE Canadian Conference on Electrical and Computer Engineering (CCECE), Regina, SK, 2013, pp. 1-5. 10.1109/CCECE.2013.6567770

[30] Alex Krizhevsky, ImageNet classification with deep convolutional neural networks, Proceeding NIPS'12 Proceedings of the 25th International Conference on Neural Information Processing Systems - Volume 1, Pages 1097-1105

[31]Shang et al. (2016) Shang W, Sohn K, Almeida D, Lee H. Understanding and improving convolutional neural networks via concatenated rectified linear units. Proceedings of 33rd international conference on machine learning (ICML2016), vol. 48; Princeton. 2016. pp. 2217-2225.

[32] M. I. Razzak and S. Naz, "Microscopic Blood Smear Segmentation and Classification Using Deep Contour Aware CNN and Extreme Machine Learning," 2017 IEEE Conference on Computer Vision and Pattern Recognition Workshops (CVPRW), Honolulu, HI, 2017, pp. 801-807.

[33] Putzu L., Ruberto C. D. White blood cells identification and counting from microscopic blood images. Proceedings of the IWBBIO; 2013; p. p. 363.

[34] Cecília Lantos, Steven M. Kornblau and Amina A. Qutub, "Chapter 5 Quantitative-Morp phological and Cytological Analyses in Leukemia", IntechOpen 2018. 